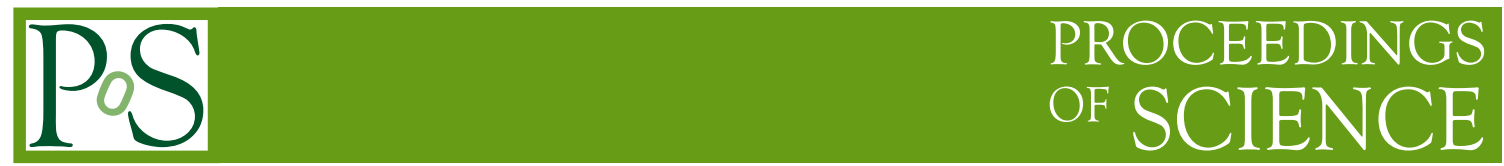

\title{
Dark Matter and Axion Searches
}

\section{Babette Döbrich ${ }^{a, *}$}

${ }^{a}$ CERN,

Esplanade des Particules 1, 1211 Geneva 23, Switzerland

E-mail: babette.dobrich@cern.ch

This contribution highlights very recent progress in two 'hot topics' of cold Dark matter direct detection: Searches via 'electronic recoil' and axion haloscope searches.

*** The European Physical Society Conference on High Energy Physics (EPS-HEP2021), ***

*** 26-30 July 2021 ***

*** Online conference, jointly organized by Universität Hamburg and the research center DESY ***

\footnotetext{
${ }^{*}$ Speaker
} 


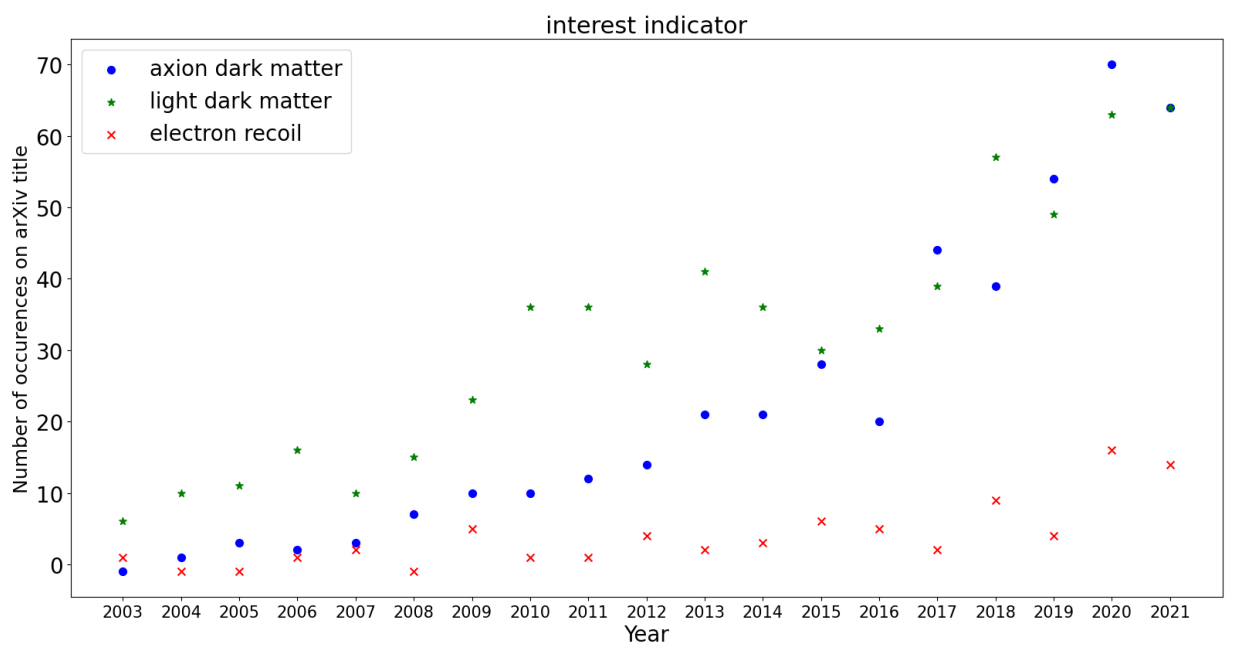

Figure 1: Number of occurrences of the search terms 'axion dark matter' (blue dots), 'light dark matter' (green asterisk) and electron recoil (red crosses) in the arxiv title, accessed in September 2021 (thus, the data-set for the year 2021 is not complete).

\section{The less-explored mass ranges}

Solving the question about the nature of Dark Matter remains the pressing question in particle physics and a broad experimental direct detection program that covers many possible decades of masses and a plethora of particle types is under way, see the APPEC report [1] for a recent discussion. This contribution cannot review the entirety of the topic suggested in its title in breath. Instead it aims to point out a selection of a few interesting, recent developments in the field. Particularly it focuses at energy scales below the typical searches above the $\mathrm{GeV}$ scale.

The first topic is direct detection via electron recoil, which gives access to studying Dark Matter at the MeV scale, often referred to as 'light dark matter' or 'light dark sector'. This is a relatively young field: even with small exposure times, sometimes orders of magnitude of sensitivity improvements are possible as backgrounds reduction and modeling are improving. The second focus lies on the direct detection of axion Dark Matter below the eV scale: Over the past few years a number of ideas to tackle the post-inflationary mass-range have finally been realized and one can continue to expect to witness a high frequency of new experimental results in this field.

Figure 1 is meant to give an illustrative indication of this development, with no claim of accuracy or completeness: It just counts the number of search term in the arxiv titles in past years for serch terms somewhat connected to the two topics that we want to discuss below. Note that the year 2021 is only considered up to the writing of these proceedings (September), so the plot does not necessarily indicate a drop in interest in 2021. 


\section{Electron recoil}

As the energy transfer for elastic nuclear recoils becomes inefficient unless the mass of the Dark Matter $m_{\mathrm{DM}} \approx m_{\text {target }}$, electron recoils become an appealing possibility when seeking to study Dark Matter at the MeV scale. By contrast, the challenge lies in the fact that in order for $\mathrm{E}_{\mathrm{DM}}$, kinetic $>E_{\text {binding, typically }} m_{\mathrm{DM}}>300 \mathrm{keV}\left(\mathrm{E}_{\text {binding }} / 1 \mathrm{eV}\right)$. Thus, detectors enabling a rather low electron recoil threshold are needed to explore $\mathrm{MeV}$-scale Dark Matter candidates. Appropriate materials can include, e.g., semiconductors, noble liquids or scintillators. Even lower thresholds can be achieved using exotic set-ups, like superconducting aluminum, but to this end, still more R\&D is needed, see e.g. [2].

In this rapidly evolving field, even couple of days of data-taking can gain orders of magnitude in sensitivity, as nicely exemplified by the recent 2020 SENSEI results [3]: Going from 0.2 g-days exposure in 2018 to about 48 g-days (plus an upgrade from prototype to science-grade detectors), allowed a more than 4 orders of magnitude sensitivity gain in probing $\sigma_{e}$ for Dark Matter masses of tens to hundreds of $\mathrm{MeV}$.

Another jump of sensitivity improvement in these experiments can be expected by a better understanding of backgrounds. Some developments $[4,5]$ as well as topical discussions [6] have taken place in the last year in this respect: Note that at the moment all limits are produced under the conservative assumption that all recorded events are indeed produced by a Dark Matter signal.

It should be highlighted that the possibility exists, that an electron recoil signal has already been seen in XENON1T [7]. The XENON1T TPC combines the so called S1 (prompt light signal) and S2 signals (secondary light from drifted charges) to gain insight on the position and energy of an event. Since the S1/S2 ratio is rather different for nuclear and electronic recoils, a dedicated search for electron recoil events was performed yielding a $\sim 3 \sigma$ excess between energies of 1-7keV.

A number of new-physics interpretations to this excess have been put forward in the last year, resulting in large compilation of fits to a possible signal [8]. The most likely SM interpretation by now is a Tritium contamination (which however cannot be confirmed or excluded by the collaboration currently). Clarification can be expected thanks to a careful materials selection and data from XENONnT in $~ 2022$ : It will also have the advantage of 4 Tons fiducial volume instead of 'only' 1 Ton.

Let us close by mentioning that electron recoil experiments can also contribute to setting Dark Photon DM limits [9], which makes them an even more versatile tool in Dark Matter physics.

\section{Axion Searches}

The 'vanilla' axion Dark Matter candidate (that solves the strong CP problem) while (yet) escaping experimental detection, lives a few orders of magnitude below the eV scale. Though recent model building efforts have shown that there is motivation to search it outside its benchmark model parameters (see e.g. [10,11]). If the model is such that the strong CP problem is not solved anymore (but the problem of dark matter could be), one mostly speaks of Axion-like particles (ALPs) instead of the axion. A recent review of axion theory and numerous models and constraints is given in [12]. Being so light-weight in the arguably most interesting parameter space, its search often necessitates techniques that might not be considered in the realm of 'high energy physics'. 
Despite (or because) of this, a plethora of new experiments have entered the field in the past few years (see [13] for a recent review on the experimental landscape of axion searches). An indication of this development is the steep increase of the number of occurrences of the search term 'axion dark matter' in titles of papers on the arxiv, shown in Fig. 1 (note that the data point for 2021 includes only submissions until September 2021. Note also that simply searching for 'axion' will yield $\gg 300$ hits in 2021!).

As with other Dark Matter candidates, one can search for it via production and consecutive detection: In the case of axions, popular methods are searches for Axions (and ALPs) produced in the sun with Helioscopes, or searches for ALPs produced via a laser beam (light shining through the walls). In the following, we focus again on direct detection.

Figure 2 summarizes experimental exclusions for axions and ALPs in the radiofrequency range. The benchmark axion model range (KSVZ and DFSZ) is indicated in yellow. In red, laboratory constraints are shown, green denote astrophysical constraints. We wish to highlight a few of the most recent searches in the following, but first want to explain the general idea behind these experiments.

Axion direct detection here proceeds through the haloscope technique (i.e. the conversion of a DM axion into photons in an RF cavity inside a magnetic field) and the figure of merit in such set-ups goes as $F \sim g_{a \gamma}^{4} m^{2} B^{4} V^{2} T_{\text {sys }}^{-2} \mathcal{G}^{4} Q$. In order to probe low couplings $g_{a \gamma}$ one thus wishes to maximize the external magnetic field $B$, the cavity resonance quality $Q$, the cavity volume $V$, whilst a geometric factor $\mathcal{G}$ is normally already maximized by design. $\mathcal{G}$ is a measure of directional overlap of the cavity electric field modes with the external magnetic field. By contrast, the system temperature $T_{\text {sys }}$ should be as low as possible. Once an envisaged target sensitivity is achieved for the axion mass channels within the cavity resonance, the cavity is usually detuned to explore the next set of mass channels. Note that a large axion mass means a large resonance frequency. Going to larger masses therefore is naively is tied to a smaller geometry and thus lesser volume, negatively impacting the reachable sensitivity. This gives a feeling for why Figure 2 displays the strongest (while widest) limits at the lowest mass range.

At the low end of the search window the most recent incarnation of ADMX has been conceived to scan 500MHz/year at DFSZ sensitivity. DFSZ axions have been excluded in 2020 through the "run 1B analysis" [15] from roughly 2.8 to $3.3 \mu \mathrm{eV}$. A preliminary analysis presented in 2021 [16] (run 1c), tackled masses up to $4.2 \mu \mathrm{eV}$, partially at DFSZ and partially at KSVZ sensitivity. With this result, ADMX will soon tackle the post-inflationary mass range (i.e. when the Peccei Quinn phase transition happens only after inflation), in which the axion mass becomes predictable 'in principle'. A number of computations have been published that attack this challenging task. For more details on this we would like to point out a nice, pedagogical review in which of these aspects and some results are summarized [17].

Going to much larger masses, there have been several recent advances to push the figure of merit overcoming the above-mentioned complications of building a competitive haloscope. For example, preparing the cavity mode in a squeezed state, it has been shown that the scanning speed can be improved by a factor of 2 [18]. Further tuning knobs are to decrease $T_{\text {sys }}$ down to the quantum limit, and very large quality factors using, e.g. superconducting materials in the cavity [19]. The latter option was also subject of a topical workshop this year [20].

Results that are based on a cavity that keeps a large resonance frequency, while not suffering from volume loss via a multi cell cavity have been built and used for data-taking at CAPP [21] and 


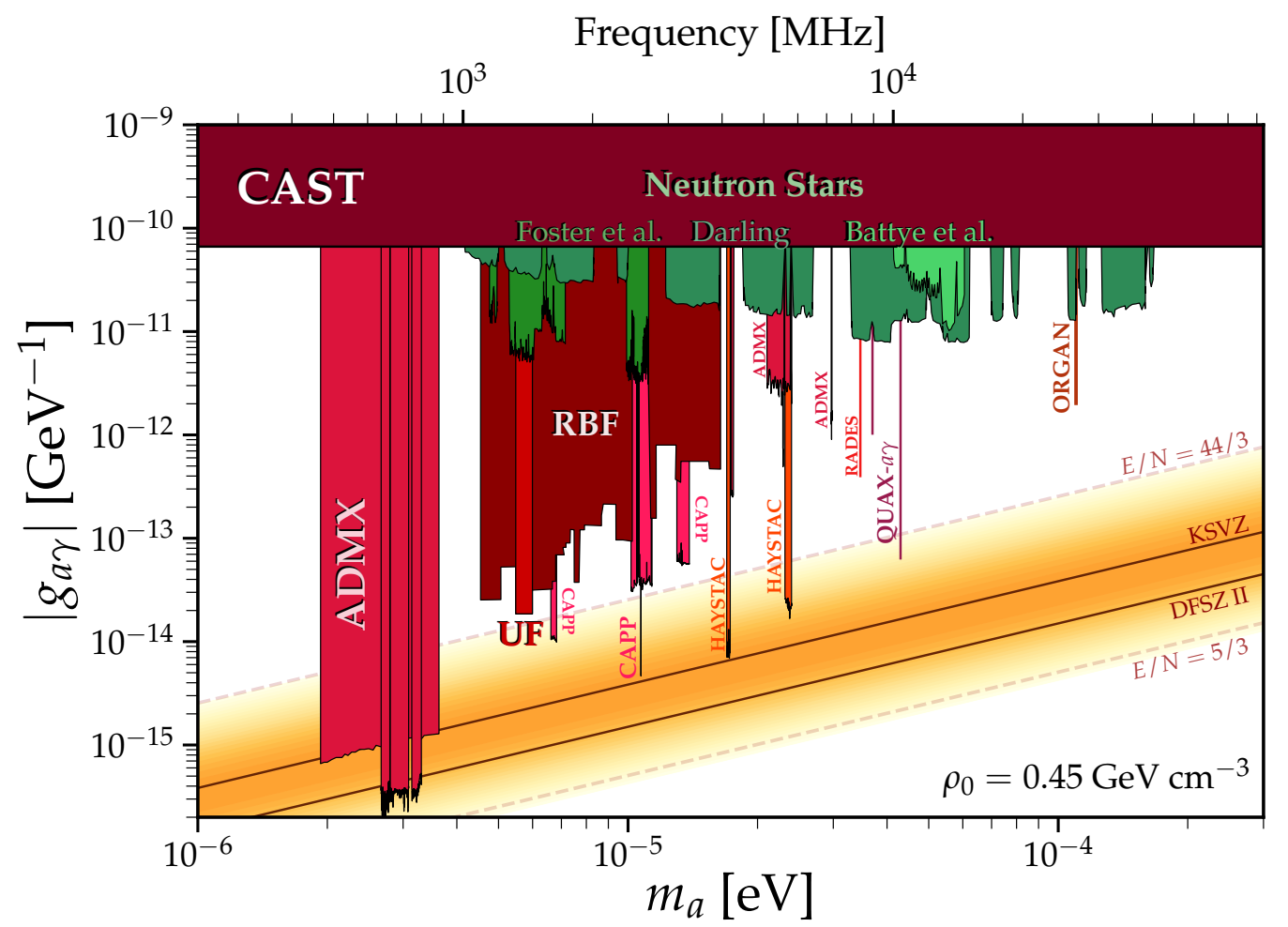

Figure 2: Overview of limits on axion-photon coupling mid-2021, taken from [14]. The yellow region indicates benchmark axion models, red are laboratory constraints. Notably, constraints drawn 'parallel to the coupling axis' usually stem from narrow band scanning haloscopes (discussed here), whereas the constraint which is flat across the mass range is due to the CAST solar axion search). Green are astrophysical constraints, which are not discussed here.

CERN [22]. Via phase-matching of multiple cavities also a larger effective volume can be reached.

In summary, in this dynamic field many new groups are helping to explore the entirety of the remaining axion parameter space shown in Fig. 2, while old-hands (such as ADMX) are able to systematically scan even to DFSZ sensitivity.

Including projections of proposed experiments and those that are under construction (see [14] for an up-to-date list), suggests that the axion will not be able too hide very long in case it exists.

\section{Conclusions}

There is plenty of viable parameter space for Dark Matter with masses well below the $\mathrm{GeV}$ scale. Recently, experimental activity is sparking in electronic recoil searches ( $\mathrm{MeV}$ scale) and axion searches via haloscopes (sub-eV scale). Besides the theory motivation of the Dark matter candidates themselves, the prospect of making a huge impact experimentally even without decades of prior R\&D work has lead to fast-pace developments in those fields during the past year. Above we highlighted a selection of these advances. 


\section{Acknowledgments}

I would like to thank the organizers for inviting me to present this summary, as well as F. Kalhoefer, T.-T. Yu, B.v. Krosigk and M. Schumann for useful discussions in preparing the talk at EPS-HEP. Also thanks to S. Meehan for his initial work on the python script used to make Figure 1. BD is supported through the European Research Council under grant ERC-2018-StG-802836 (AxScale project).

\section{References}

[1] J. Billard, M. Boulay, S. Cebrián, L. Covi, G. Fiorillo, A. Green, J. Kopp, B. Majorovits, K. Palladino and F. Petricca, et al. [arXiv:2104.07634 [hep-ex]].

[2] M. Battaglieri, A. Belloni, A. Chou, P. Cushman, B. Echenard, R. Essig, J. Estrada, J. L. Feng, B. Flaugher and P. J. Fox, et al. [arXiv:1707.04591 [hep-ph]].

[3] L. Barak et al. [SENSEI], Phys. Rev. Lett. 125, no.17, 171802 (2020) doi:10.1103/PhysRevLett.125.171802 [arXiv:2004.11378 [astro-ph.CO]].

[4] P. Du, D. Egana-Ugrinovic, R. Essig and M. Sholapurkar, [arXiv:2011.13939 [hep-ph]].

[5] L. Barak, I. M. Bloch, A. Botti, M. Cababie, G. Cancelo, L. Chaplinsky, F. Chierchie, M. Crisler, A. Drlica-Wagner and R. Essig, et al. [arXiv:2106.08347 [physics.ins-det]].

[6] Excess workshop: https://indico.cern.ch/event/1013203/

[7] E. Aprile et al. [XENON], Phys. Rev. D 102, no.7, 072004 (2020) doi:10.1103/PhysRevD.102.072004 [arXiv:2006.09721 [hep-ex]].

[8] https://twitter.com/OzAmram/status/1277609718085816326

[9] A. Caputo, A. J. Millar, C. A. J. O’Hare and E. Vitagliano, [arXiv:2105.04565 [hep-ph]].

[10] P. Agrawal, J. Fan, M. Reece and L. T. Wang, JHEP 02, 006 (2018) doi:10.1007/JHEP02(2018)006 [arXiv:1709.06085 [hep-ph]].

[11] L. Di Luzio, F. Mescia and E. Nardi, Phys. Rev. Lett. 118, no.3, 031801 (2017) doi:10.1103/PhysRevLett.118.031801 [arXiv:1610.07593 [hep-ph]].

[12] L. Di Luzio, M. Giannotti, E. Nardi and L. Visinelli, Phys. Rept. 870, 1-117 (2020) doi:10.1016/j.physrep.2020.06.002 [arXiv:2003.01100 [hep-ph]].

[13] I. G. Irastorza and J. Redondo, Prog. Part. Nucl. Phys. 102, 89-159 (2018) doi:10.1016/j.ppnp.2018.05.003 [arXiv:1801.08127 [hep-ph]].

[14] Ciaran O'HARE. (2020). cajohare/AxionLimits: AxionLimits (v1.0). Zenodo. //doi.org/10.5281/zenodo.3932430 
[15] C. Bartram et al. [ADMX], Phys. Rev. D 103, no.3, 032002 (2021) doi:10.1103/PhysRevD.103.032002 [arXiv:2010.06183 [astro-ph.CO]].

[16] T. Nitta, these proceedings

[17] I. G. Irastorza, [arXiv:2109.07376 [hep-ph]], contributed to the 'Les Houches School on Dark Matter 2021' lecture notes

[18] K. M. Backes et al. [HAYSTAC], Nature 590, no.7845, 238-242 (2021) doi:10.1038/s41586021-03226-7 [arXiv:2008.01853 [quant-ph]].

[19] A. Rettaroli [Quax], Nuovo Cim. C 43, no.2-3, 93 (2020) doi:10.1393/ncc/i2020-20093-4

[20] PBC technology Miniworkshop: https://indico.cern.ch/event/1057715/

[21] J. Jeong, S. Youn, S. Bae, J. Kim, T. Seong, J. E. Kim and Y. K. Semertzidis, Phys. Rev. Lett. 125, no.22, 221302 (2020) doi:10.1103/PhysRevLett.125.221302 [arXiv:2008.10141 [hep-ex]].

[22] A. Á. Melcón et al. [CAST], [arXiv:2104.13798 [hep-ex]], accepted for publication in JHEP 